九州大学学術情報リポジトリ

Kyushu University Institutional Repository

\title{
Three-dimensional strain mapping using in situ $X$-ray synchrotron microtomography
}

Toda, Hiroyuki

Toyohashi University of Technology

Maire, E.

Universite de Lyon

Aoki, Yoshimitsu

Electronics \& Electrical Engineering, Keio University

Kobayashi, Masakazu

Toyohashi University of Technology

ht tp://hdl. handle. net/2324/1812236

出版情報: Journal of Strain Analysis for Engineering Design. 46 (7), pp.549-561，2011-07-29 バージョン：

権利関係 : 
Three-dimensional strain mapping using in-situ X-ray synchrotron microtomographFy

H Toda $^{*}$, E Maire $^{* *}$, Y Aoki $^{* * *}$, and M Kobayashi ${ }^{*}$

Department of Mechanical Engineering, Toyohashi University of Technology,

Toyohashi, Aichi, Japan

* Department of Mechanical Engineering, Toyohashi University of Technology,

Toyohashi, Aichi 441-8580, Japan (e-mail: toda@me.tut.ac.jp, tel: +81-532446697, fax:

$+81-532446690($ H. Toda))

** Universite de Lyon, INSA-Lyon, MATEIS CNRS UMR5510, 7 avenue Jean Capelle,

F-69621 Villeurbanne, France 
*** Electronics \& Electrical Engineering, Keio University, Yokohama, Kanagawa 223-

8522, Japan

Abstract: Recent advances in X-ray microtomography provide remarkable tools to image the interior of materials. Microstructural images that are similar to or about 1 order of magnitude higher than those currently attained with light microscopy can now be obtained in three-dimensions with the synchrotron radiation facilities. Local strain mapping is readily enabled by processing such high-resolution tomographic images either by the microstructural tracking technique or the digital volume correlation technique. This article is a digest of our recent research activities on three-dimensional strain mapping and also provides information on methodology. Recent applications to various issues in the materials science and engineering are also demonstrated. Possible future directions are finally discussed.

Keywords: Computed tomography; microstructural tracking; digital volume correlation; local strain mapping; 3D

\section{Introduction}

Continuum assumption provides reasonable approximation in characterizing the constitutive behaviour of materials. Local plastic strain, however, deviates from the 
continuum assumption in real materials as local deformation is influenced by the existence of various secondary phases and microscopic defects such as voids and cracks. Heterogeneity also arises in polycrystalline materials due to deformation mismatch among neighbouring grains with different crystallographic orientations.

Cross-sectional observation has been used extensively to obtain local strain distribution. Typical approaches for it would be the grid strain analysis [1] and the in-situ stereoimaging [2]. A surface observation, however, reflects surface-specific twodimensional (2D) deformation behaviour, which is often significantly different from that in the interior due to stress relaxation at a free surface. In fact, it has been claimed that the extent and growth rate of particle damage are significantly different between the surface and the bulk of strained materials $[3,4,5]$. The validity of such in-situ surface observations with respect to the bulk damage mechanisms has to be, therefore, checked with caution. A further general drawback of a cross-sectional observation is extensive deformation and damaging of a matrix and secondary phases during metallographic sample preparation that are located on specimen surface. For example, circular particles observed on a cross-section are, as a matter of fact, hemispherical that have been ground during a sample preparation process. Pores/voids are also easily filled with abrasive powders during a sample preparation process [6]. Furthermore, complicated microstructural features do not allow cross-sectional observations to reveal the threedimensional (3D) character of internal microstructure. It should be, therefore, noted that phenomena themselves observed on a specimen surface to be analyzed with local strain 
mapping can be surface-specific artefacts.

In-situ X-ray microtomography (XMT) provides a unique possibility to access the 3D character of internal microstructure and its time evolution behaviours non-destructively, thereby enabling advanced techniques for measuring local strain distribution. Recently some attempts have been made to achieve this with reasonable spatial resolution and a relatively large specimen size utilizing synchrotron radiation facilities $[6,7,8,9,10,11]$. The techniques can be classified into two types; a microstructural tracking technique [6, $7,8,9]$ and a digital volume correlation technique [12, 13]. With these techniques, 3D strain fields can be measured with reasonable accuracy [9]. Even local crack driving forces (LCDF), such as local variations in the stress intensity factor and $\mathrm{J}$ integral along a crack front line, can be measured from discrete crack tip displacement fields $[7,14$, 15]. In the present paper, principles, methods and techniques relevant to $3 \mathrm{D}$ local strain measurement will be described, together with applications to various issues of the materials science and engineering scientific domains.

\section{Principles, methods and techniques of 3D local strain mapping}

The technique for reconstructing the spatial distribution of the X-ray attenuation coefficient is known as computed tomography (CT). The third-generation synchrotron radiation (SR) facilities, such as the ESRF and SPring-8, have been identified as ideal X-ray sources for CT, providing a high-energy X-ray beam with excellent monochromaticity. CT performed at the SR facilities has enabled the reconstruction of 
volumes with a typical spatial resolution of about $1 \mu \mathrm{m}[16]$. X-ray imaging microscopy with an X-ray objective and zoom synchrotron tomography $[17,18]$ with a divergent beam provide two alternatives for the observation of microstructural features, enabling even higher spatial resolution at a 100 nm-level $[19,20,21,22]$. In addition to the ordinary 3D observations, in-situ XMT observations have been performed during loading by utilizing specially designed test rigs and a miniaturized specimen that are mounted on a rotation stage for XMT [5, 23, 24, 25, 26]. Owing to the high-resolution optical set-ups, microstructural features and their time evolution behaviours have been clearly visualized in various engineering materials [7, 14, 19, 23, 25, 27, 28, 29].

In the field of the velocimetry, particle tracking velocimetry is known as a method by which the velocity of fluids is measured in two-dimensional optical images of a flow [30]. The paths of tracer particles are reconstructed by matching each pair of particles in consecutive frames with an appropriate trajectory prediction method, taking differences in size, location, shape etc. into account. Although it is not so straightforward, the extension of these techniques to 3D tomographic images has been reported recently [6, $7,8,9]$.

In order to perform 3D microstructural tracking, all the microstructural features, such as particles and pores, visible in reconstructed CT images are segmented and labelled. Morphological characteristics of each microstructural feature, e.g., surface area, volume and gravity centre, are measured precisely. A high-resolution 3D surface construction algorithm, such as the marching cube algorithm, which gives a pentagonal faceted iso- 
intensity surface, has been often used to improve accuracy to a sub-voxel level [31]. Fig. 1 shows a schematic illustration of image registration process. Since strain is calculated from the physical displacement of each microstructural feature that is observed at two consecutive loading steps, precise image registration between a pair of in-situ XMT images is of crucial importance for accurate strain mapping. For example, in the present authors' research, translational and rotational shifts between a pair of in-situ XMT images are minimized so that the sum of differences in distance between corresponding marker pairs is minimized after roughly performing registration using eight relatively coarse markers located near the corners of a field of view [9].

Microstructural tracking mostly depends on the estimate of particle centroids in image frames. Various efforts have been made to raise accuracy to a reasonable extent. For instance, the concept of cluster matching has been applied by Kobayashi et al., and it has been found to perform well in the presence of microstructural agglomeration. Nakazawa et al., have proposed a landscape method by which image registration can be skipped by making a tracking process in two steps. In the following sections, the different approaches for microstructural tracking are reviewed.

\subsection{General two-frame tracking}

Nielsen et al. and Kobayashi et al. have used similar two-frame tracking methods to solve the correspondence problem occurring in microstructural tracking [8,9]. Nielsen et al. have used the centre of gravity and volume of each particle [8], whereas 
Kobayashi et al. have used surface area in addition to the two parameters in order to utilize complexity in particle shape [9]. Kobayashi et al. have introduced the following matching probability parameter (MPP), $M_{\mathrm{p}}$, to suppress tracking errors;

$$
M_{\mathrm{P}}=\alpha L_{i, j}+\beta S_{i, j}+\gamma V_{i, j}
$$

Here $L_{i, j}, S_{i, j}$ and $V_{i, j}$ are the centre of gravity, surface area and volume of a particle, respectively, and defined so that they vary between 0 and 1 in a pre-determined search domain in which a corresponding marker is searched. $\alpha, \beta$ and $\gamma$ are weights, where $\alpha+\beta+\gamma=1$. Index numbers, $i$ and $j$, denote feature numbers which are assigned in the labelling process. Firstly one microstructural feature is picked up from a dataset, and then $M_{\mathrm{p}}$ values for all the markers in a corresponding dataset are calculated using eq. (1). If the maximum value of $M_{\mathrm{p}}$ is larger than a pre-determined first threshold value, $M_{\mathrm{p}}^{\text {th1 }}$, and a difference between the maximum and the second largest values is larger than another pre-determined threshold value, $M_{\mathrm{p}}^{\text {th2 }}$, then the marker pair with the largest $M_{\mathrm{p}}$ value is labelled to be tracked. If the difference is less than $M_{\mathrm{p}}^{\text {th2 }}$, then the marker pair is held in a waiting list. If no marker satisfies the first criterion on $M_{\mathrm{p}}^{\text {th1 }}$, the marker is not used for the tracking purpose.

\section{2 Cluster matching}

Kobayashi et al. [9] have applied another tracking algorithm called modified spring model algorithm (SMA) [32]. It is a two-frame tracking method to perform pattern matching for particle clusters. The particles are assumed to be connected by invisible 
elastic springs. This method identifies particle pairs by searching for the smallest total spring energy among particle clusters, in which particles are connected by imaginary springs to each other. The total elastic energy for imaginary springs, $E_{\mathrm{sp}}$ is given as follows;

$$
E_{\mathrm{sp}}=\frac{1}{N_{\mathrm{sp}}} \sum_{i=1}^{N_{\mathrm{sp}}} \frac{\left|b_{i}^{\prime}-b_{i}\right|}{\left|b_{i}\right|}
$$

where $N_{\mathrm{sp}}$ is the number of imaginary springs connecting particles. $\mathbf{b}_{i}$ and $\mathbf{b}_{i}^{\prime}$ are relative vectors calculated from marker positions before and after deformation, respectively. The method would be effective for locally inhomogeneous deformation, because SMA can cope with apparently random deviation of relative position among particles. Kobayashi et al. have combined SMA with MPP to minimize markers pended in the MPP process that are agglomerated with the other tracked and pended markers. It has been concluded that this algorithm is efficient in tackling unpaired particles with MPP, resulting in an improved confidence pertaining to the obtained strain distribution.

\subsection{Trajectory prediction}

Trajectory predictions have been also applied to track particles in conjunction with the MPP method [9, 15]. Provided that strain distribution is homogeneous throughout a material, the centre of gravity coordinate of each marker in the image dataset of a deformed sample is linearly interpolated before applying the MPP method described above. When a model sample is monotonously tensioned, image datasets are extended 
only in the longitudinal, i.e. tensile, direction and contracted in the vertical directions assuming Poisson's ratio of 0.5 [9]. Another example is the application to a cracked medium performed by $\mathrm{Qu}$ et al. [15], in which the best-match particle pairs were determined after distorting one image by applying a combination of a linear elastic neartip displacement field and a non-linear elastic-plastic near-tip displacement field called the HRR field. The boundary of the two displacement fields was assumed to be a plastic zone size. With the particle trajectory prediction, Qu et al. have reported that more than $75 \%$ of particles $(6,973$ particles) could be tracked successfully, which seems to be sufficient to calculate local crack driving force distribution along a crack front line.

Multi-frame trajectory prediction has been also applied to inhomogeneous plastic deformation [33]. Kobayashi et al. have used the following polynomial to extrapolate marker locations for two to three loading steps to predict a marker position in the subsequent loading step.

$$
p(x)=a_{n} x^{n}+a_{n-1} x^{n-1}+\cdots+a_{2} x^{2}+a_{1} x+a_{0} .
$$

where $x$ is a coordinate and $a_{\mathrm{k}}$ is a coefficient to be calculated. The estimate of a trajectory involves findings all the coefficients in eq. (3). Once the coefficient vector is found, computing the location at the subsequent loading step is straightforward. Although time consuming, this algorithm seems more efficient for inhomogeneous deformation that cannot be described with conventional constitutive equations. Particles which are tracked in this manner have superior probabilities of successful matching in a multi-frame succession that is very nearly equal to $100 \%$ [33]. 
Overall, compared to the conventional estimate of particle centroids, the trajectory prediction techniques increase the spatial resolution and measurement accuracy significantly.

\subsection{Tracking for invisible features}

When feature A becomes invisible at step $n$, the physical displacements between steps $n-1 \sim n$ of particles that are located within a search area of $R$ in radius can be used to predict an invisible trajectory for feature A. Scattered data interpolation of this type is achieved using radial basis functions (RBF) [29], which are circularly symmetric functions centred at particular points. RBFs can provide interpolants to function values given at irregularly positioned points, providing excellent approximations to the underlying function, even when the number of interpolation points, $N$, is small. In this case, the function and interpolation points correspond to a displacement field and particles in the matrix, respectively. RBFs may be used to interpolate a function with $N$ points by using $N$ RBFs centred at these points as follows.

$$
u^{i}=\sum_{j=1}^{N} c^{j} \cdot g\left(\left\|p_{i}-p_{j}\right\|\right)
$$

where $u^{i}$ is particle displacement during macroscopic compression, $\|$.$\| is the Euclidean$ distance between particles, $g(\|\|$.$) is the radial functions, and c^{j}$ is the interpolation coefficient to be determined. To solve for $c^{j}$, the following system of linear equations must be solved. 


$$
\left(\begin{array}{ccc}
u_{x}^{1} & \cdots & u_{x}^{N} \\
u_{y}^{1} & \cdots & u_{y}^{N} \\
u_{z}^{1} & \cdots & u_{z}^{N}
\end{array}\right)=\left(\begin{array}{ccc}
c_{x}^{1} & \cdots & c_{x}^{N} \\
c_{y}^{1} & \cdots & c_{y}^{N} \\
c_{z}^{1} & \cdots & c_{z}^{N}
\end{array}\right)\left(\begin{array}{ccc}
g\left(\left\|p_{1}-p_{1}\right\|\right) & \cdots & g\left(\left\|p_{1}-p_{1}\right\|\right) \\
\cdots & \cdots & \cdots \\
g\left(\left\|p_{N}-p_{1}\right\|\right) & \cdots & g\left(\left\|p_{N}-p_{N}\right\|\right)
\end{array}\right)
$$

By choosing an appropriate radial basis function, such as the Gaussian, the matrix is obviously positive-definite real symmetric. Thus, a solution always exists to this system of equations. Once $c^{j}$ is obtained, the physical displacement of an invisible feature, $u$, which is located at the centre of a search area, is predicted as follows.

$$
u=\sum_{i=1}^{N} c^{j} \cdot g\left(\left\|P-p_{i}\right\|\right)
$$

where $P$ is the initial location of the feature.

Toda et al. have applied this technique to pore re-initiation behaviours during hightemperature exposure after annihilation during rolling. Each pore that has been initiated around a disappeared pore is judged to be re-initiated [29].

\subsection{Hierarchical tracking procedure}

To match more than ten thousand dispersed particles, a hierarchical tracking method has been suggested [34]. In this approach, a certain number of largest particles are regarded as landmarks to gain approximate displacement of the material. The rest is regarded as non-landmarks. They are matched by displacement vector estimation based on landmark results by using RBF transformation.

\section{Relaxation method for landmark matching}

Relaxation method [35] is employed to match the landmarks during deformation. In 
this method, renewing a probability matrix optimizes the matching relations of the landmarks while considering the conformity of neighbourhoods. Namely, the probability matrix is renewed under the assumption that neighbourhoods must be able to be matched if the matching relation of a certain landmark is correct.

If the sets of landmarks before and after deformation, the number of which are $N$ and $M$ respectively, are defined as $A=\left\{a_{1}, a_{2}, \ldots, a_{N}\right\}$ and $B=\left\{b_{1}, b_{2}, \ldots, b_{M}\right\}$, then the probability vector between $a_{i}$ and $B$ is expressed as $p_{i}=\left[p_{i(1)}, p_{i(2)}, \ldots, p_{i(M)}\right]^{T} \hat{I} R^{M}$. The probabilities are always positive and satisfy $\sum_{k^{\prime}} p_{i\left(k^{\prime}\right)}=1$. The set of $N$ probability vectors constitutes the probability matrix between $A$ and $B \boldsymbol{p}=\left[\boldsymbol{p}_{1}, \boldsymbol{p}_{2}, \ldots, \boldsymbol{p}_{N}\right]^{T} \in R^{N \times M}$.

\section{Initialization of the probability matrix}

If the volume change between $a_{i}$ and $b_{k}$ is markedly great, then $p_{i(k)}$ should be zero; otherwise, it is given the same probability as the others (7).

$$
\begin{gathered}
p_{i(k)}^{0^{\prime}}= \begin{cases}1 & \text { if }\left|\frac{V_{k}-V_{i}}{V_{i}}\right| \leq R C \\
0 & \text { otherwise }\end{cases} \\
p_{i(k)}^{0}=\frac{p_{i(k)}^{0^{\prime}}}{\sum_{k^{\prime}} p_{i\left(k^{\prime}\right)}^{0^{\prime}}}
\end{gathered}
$$

where $V_{i}$ and $V_{k}$ are the volume of $a_{i}$ and of $b_{k}$, respectively. Additionally, we define the allowable volume change $R C$ in order to ignore the matching relations which have 
markedly great volume changes.

\section{Renewal of the probability matrix}

$p_{i(k)}$ is renewed by the renewal coefficient $q_{i(k)}$ as shown in (9).

$$
\begin{gathered}
p_{i(k)}^{t+1}=\frac{q_{i(k)} p_{i(k)}^{t}}{\sum q_{i\left(k^{\prime}\right)} p_{i\left(k^{\prime}\right)}^{t}} \\
q_{i(k)}=\sum_{j \in \zeta_{i}} \max \left(\gamma_{i j}(k, l) p_{j(l)}^{t}\right) \\
g_{i j}(k, l)=\max \left(1-\frac{1}{W} e_{i j}(k, l), 0\right) \\
e_{i j}(k, l)=\frac{\left\|d_{k l}-d_{i j}\right\|}{\left\|d_{i j}\right\|}
\end{gathered}
$$

$q_{i(k)}$ represents the conformity with the neighbourhood set of $a_{i}$, provided that $a_{i}$ matches $b_{k}$. To define the neighbourhood set $\zeta_{i}$, Quickhull [36] constructs the undirected graphs $G$ by generating Delaunay tetrahedrons from $A$. Then, let $\zeta_{i}$ denote $\left\{a_{j^{\prime}} \mid\left[a_{i}, a_{j^{\prime}}\right] \in G, i \neq j^{\prime}\right\}$. Among the set of landmarks after deformation $B$, the likeliest-matching candidate $b_{l}$ of a neighbourhood $a_{j}$ is the one which maximizes the product of $\gamma_{i j}(k, l)$ and the present probability $p_{j(l)}^{t}$. As shown in (10), $q_{i(k)}$ is obtained by summing the above product of each neighbourhood. Here, $\gamma_{i j}(k, l)$ is the evaluation function of matching $a_{i} \leftrightarrow b_{k}$ and $a_{j} \leftrightarrow b_{l}$. This function is a decreasing function, which is calculated from the ratio of expansion and contraction $e_{i j}(k, l)$ in (12). As shown in 
(11), if $e_{i j}(k, l)$ is $0, \gamma_{i j}(k, l)$ becomes 1 as the maximum value. On the other hand, when $e_{i j}(k, l)$ is over the worst ratio $W$ given in advance, $\gamma_{i j}(k, l)$ becomes 0 .

If the sum of the absolute differences of probabilities $\Delta_{i}=\sum_{k^{\prime}}\left|p_{i\left(k^{\prime}\right)}^{t+1}-p_{i\left(k^{\prime}\right)}^{t}\right|$ is below the threshold $\delta, \boldsymbol{p}_{i}$ can be considered to be converged. $a_{i}$ matches the one which has the highest probability in the set of landmarks after deformation $B$.

\section{Non-landmark matching}

The displacement vectors of non-landmarks are estimated by those of landmarks. The typical method is RBFT, which is explained in 2. 4 .

\subsection{Strain mapping using tracked markers}

When a sufficient number of many markers are dispersed in 3D space, the Delaunay tessellation algorithm [36], which generates an aggregate of space-filling irregular tetrahedral, is used to obtain local strain distribution. The vertices of each simplex define objectively four nearest neighbour marker microstructural features. Local strain is calculated in each tetrahedron, assuming a linear displacement field inside tetrahedrons. Normal strains, $\varepsilon_{x}, \varepsilon_{y}$ and $\varepsilon_{z}$ and shear strains, $\gamma_{x y}, \gamma_{y z}, \gamma_{z x}$, are calculated from displacements, $u_{i}, v_{i}, w_{i}, u_{j}, v_{j}, w_{j}, u_{k}, v_{k}, w_{k}, u_{l}, v_{l}, w_{l}$, at tetrahedron vertices $i, j, k, l$ in a rectangular coordinate system, assuming a linear displacement field inside it as follows;

$$
\left\{\varepsilon_{x} \varepsilon_{y} \varepsilon_{z} \gamma_{x y} \gamma_{y z} \gamma_{z x}\right\}^{\mathrm{t}}=\left[\mathbf{B}_{i} \mathbf{B}_{j} \mathbf{B}_{k} \mathbf{B}_{l}\right]\left\{u_{i} v_{i} w_{i} u_{j} v_{j} w_{j} u_{k} v_{k} w_{k} u_{l} v_{l} w_{l}\right\}^{\mathrm{t}}
$$


where $\left[\mathbf{B}_{i}\right]$ is given as

$$
\begin{gathered}
{\left[\mathbf{B}_{i}\right]=\frac{1}{6 V}\left[\begin{array}{ccc}
o_{i} & 0 & 0 \\
0 & p_{i} & 0 \\
0 & 0 & q_{i} \\
p_{i} & o_{i} & 0 \\
0 & q_{i} & p_{i} \\
q_{i} & 0 & o_{i}
\end{array}\right]} \\
o_{i}=y_{k} z_{j}+y_{l} z_{k}+y_{j} z_{l}-y_{k} z_{l}-y_{j} z_{k}-y_{l} z_{j} \\
p_{i}=x_{l} z_{j}+x_{j} z_{k}+x_{k} z_{l}-x_{j} z_{l}-x_{l} z_{k}-x_{k} z_{j} \\
q_{i}=x_{l} y_{k}+x_{j} y_{l}+x_{k} y_{j}-x_{j} y_{k}-x_{k} y_{j}-x_{l} y_{k}
\end{gathered}
$$

where $V$ is tetrahedron volume, $x_{j}, y_{j}, z_{j}, x_{k}, y_{k}, z_{k}, x_{l}, y_{l}, z_{l}$ indicate the vertex positions of $j, k$ and $l$ in a tetrahedron. Shifting indices such as $i$ to $j, j$ to $k, k$ to $l$ and $l$ to $i$ brings $\left[\mathbf{B}_{j}\right],\left[\mathbf{B}_{k}\right]$ and $\left[\mathbf{B}_{l}\right]$.

\subsection{Digital volume correlation}

As shown in the preceding sections, local strain measurements require that the displacement of the position of some parts of the sample due to the application of the stimulus should be measured. Instead of tracking the centre of mass of some features in the microstructure as presented earlier, it is also possible to track the displacement of recognizable patterns. A pattern is here defined as a subset of the volumic image of the sample. Ideally, this gray level distribution in the reference subvolume should be similar to the corresponding one in the deformed tomogram.

The best way of recognizing the position of a given pattern from one deformation stage 
to the next (if this can be achieved, then $u$ can be retrieved) is by correlating the two images (Digital Image Correlation -DIC). Although DIC is now extensively used in 2D with optical or SEM image as inputs (see [12] for a review), its extension to 3D with tomograms as inputs, also called Digital Volume Correlation (DVC, see [13] for a review) is quite new and still not widely used. The first example of this sort of treatment was published in [10]. Applied to bones in particular, this initial study was developed in subsequent articles and the correlation method slightly improved. In 2D as well as in 3D, the displacement measurement step involves minimization of an objective function that quantifies the match between original $(f(x))$ and trial subvolumes $g(x+v)$ with respect to a set of affine transformation parameters. A least-square function of the following type is typically used :

$$
\Phi(v)=\oiiint[g(x+v)-f(x)]^{2} d x
$$

$\Phi$ being equal to 0 when $v=u$.

The amount of published studies has slightly increased recently, thanks to the availability of new algorithms able to be interfaced with a finite element framework from the correlation point of view [11]. A review of some of the applications using DVC is given in section 3.4 .

\subsection{Accuracy of strain measurement}

To the best knowledge of the authors, the microstructural tracking and DVC haven't been quantitatively compared on a same data set so far. 
In the case of the microstructural tracking, it is reasonable to assume that strain measurement accuracy fundamentally depends on the accuracy of gravity centre measurement and inter-particle spacing. High-density strain mapping is realised by tracking as many particles as possible, which inevitably decreases the accuracy of strain mapping. As a typical situation, 30,000 particles are assumed to be uniformly dispersed in the standard-sized specimen $(600 \times 600 \times 600 \mu \mathrm{m})$ for the synchrotron XMT observation, forming a simple cubic lattice structure. Inter-particle spacing is about 20 $\mu \mathrm{m}$ in this case, resulting in the possible maximum error for strain measurement of $5 \%$ on the assumption that the special resolution of $3 \mathrm{D}$ images is close to the theoretical resolution of the projection-type XMT (i.e., about $1 \mu \mathrm{m}$ ). It has been however reported that substantial error is significantly smaller than that of the spatial resolution [37]. For example, Weckenmann et al. have measured a spacing $(20 \mathrm{~mm})$ between spheres of 2.5 $\mathrm{mm}$ in diameter using a laboratory XMT apparatus. As a result the entire measurement results were expressed within $\pm 23.6 \mu \mathrm{m}$, which corresponds to $13.4 \%$ of the theoretical resolution (twice as large as a voxel size) of $176 \mu \mathrm{m}$. Considering that the substantial spatial resolution for a laboratory XMT is usually much worse than a theoretical resolution, it can be expected that a substantial error for strain measurement falls well below $0.5 \%$. On the other hand, strain measurement accuracy also depends on the spatial frequency of recognizable pattern in DVC. Various complex factors, such as errors for pattern recognition and change in the pattern itself with global loading, might affect the strain measurement accuracy in addition to the above factors. 


\section{Recent applications of local strain mapping to the materials science and engineering}

Owing to the recent development of synchrotron XMT, which readily enables the visualization and tracking of individual microstructural features, 3D local strain measurements have been occasionally reported to date. Present space limitations require, however, that the current literature be distilled. The present section encompasses three topics with emphasis on the tracking-based methods rather than the digital image correlation, focusing on some recent original results by the present authors.

\subsection{Local strain mapping during deformation}

Shrinkage and annihilation behaviours of hydrogen pores $(1-10 \mu \mathrm{m}$ in diameter) were observed in 3D during hot and cold rolling of an Al-Mg alloy [29]. It was examined, by exposing the material to a high temperature after plastic deformation, whether complete healing of pores is achieved after plastic deformation. Although pores generally show shrinking and closing, consecutive inspection of a single specimen revealed a variety of geometrically variable behaviours. Some of the pores were reinitiated in positions identical to those before their annihilation, even after an $8 \sim 22 \%$ macroscopic strain had been further applied after annihilation. This was analyzed by utilizing the technique introduced in section 2.4. We attributed such local variations to significant local strain variation, which we measured in a series of tomographic volumes by tracking 
microstructural features. Local strain distribution is demonstrated in the form of a 3D colour contour map as shown in Fig. 3. It is interesting to note that although the macroscopic applied load is compressive, regions locally exposed to tension are seen in Fig. 3. Even in the compressive regions, compressive strain, $\varepsilon_{\mathrm{y}}$, varied widely between 0 $\sim 1.3$.

Similar annihilation behaviour of pores during surface cold working was continuously observed and analyzed by tracking artificially dispersed particles in a cast aluminium alloy, thereby mapping local strain distributions in high-density [38]. Fig. 4 shows the shrinkage and annihilation behaviours of a pore together with local strain distributions around the pore shown on a virtual cross-section. The pore was completely annihilated after $420 \mathrm{~s}$ of surface hardening treatment, reflecting the local deformation of the aluminium matrix around it as shown by the local strain distributions shown in Fig. 4. It could be inferred that micro pore annihilation requires the widespread presence of an apparent effective strain exceeding roughly $50 \%$ around the micro pores. It has been, however, hard to assess the relationship between pore closure and local strain with conclusive evidence due to the difficulty in the direct experimental measurement of 3D strain with the conventional experimental approaches. In order to specify a reasonable local requirement for pore closure, volume change of each pore is plotted as a function of $\varepsilon_{\mathrm{m}}, \varepsilon_{\mathrm{eff}}$ and $\varepsilon_{\mathrm{m}} / \varepsilon_{\mathrm{eff}}$ in Fig. 5 [38], in which both of the above-mentioned results for monotonic compression and surface cold working are plotted together. It was clarified that the magnitude and sign of hydrostatic strain, $\varepsilon_{\mathrm{m}}$, could not be associated with the 
shrinkage of a pore. The pore volume change was found to be a function primarily of the local effective strain, $\varepsilon_{\text {eff }}$, as clearly shown in Fig. 5. The complete annihilation of a pore, appears to be realized at $\varepsilon_{\mathrm{eff}} \approx 0.4 \sim 0.5$.

\subsection{Grain boundary tracking technique}

Some of the present authors (HT and MK) have proposed a new experimental method, grain boundary tracking technique [39] by which crystallographic deformation behaviour is visualized by combining the above-mentioned microstructural tracking technique and the visualization of grain boundary network [17]. The latter can be realized by decorating grain boundary with a dissimilar element having an X-ray absorption coefficient different from a matrix. In the case of aluminium alloys, we usually use gallium for grain boundary visualization [40].

In the grain boundary tracking technique, in-situ X-ray microtomography (XMT) is, first of all, performed during loading by utilizing a material test rig mounted on a rotation stage. A dopant is then applied to the specimen just before fracture in order to visualize grain boundaries in $3 \mathrm{D}$. All the visible microstructural features are then segmented and labelled in the images captured before gallium application as is so in the above-mentioned microstructural tracking technique. A 3D mask is also produced from the decorated grain boundary image. After a precise image registration, all the microstructural features are classified into grain boundary features and those located inside each grain by utilizing the grain boundary mask. The tessellation technique is 
used for grain boundary representation purpose, where all the grain boundary features extracted constitute polygonal boundary among grains. Non-regular triangles are used for this. By tracking grain boundary microstructural features in reverse chronological order, the deformation behaviour of each grain is readily reconstructed as is shown in Fig. 6. Local strain mapping during deformation is obtained inside each grain as is shown in section 3.1. We are now devoting efforts to combining the grain boundary tracking technique with the X-ray diffraction to measure the crystallographic orientation of each grain visualized with this technique [18, 41, 42].

\subsection{Comparison with image-based simulation}

As has been demonstrated in the present paper, real materials are so inhomogeneous and complex that the conventional modelling works do not provide proper interpretation at microstructural scales. Accordingly, a 3D model that takes into account real microstructural features is expected to identify the micro-mechanical behaviours of materials. The high-resolution XMT technique has been applied to create 3-D finiteelement simulation models by converting volume tomographic data sets into stereolithography tessellation language models. For example, particle damage behaviour has been investigated numerically, especially focusing on the effects of relatively small particles in the vicinity of crack-tip in an Al-7\%Si alloy, as shown in Fig. 7 (b) $[27,43]$. In this calculation, particles were embedded only in a tiny region of interest $(80 \times 80 \times 30$ $\mu \mathrm{m})$ in order to reduce computation time to a reasonable extent, whereas the 
surrounding region was homogenized using the stress-strain behaviour of the alloy. These results are in qualitative agreement with the experimental observation shown in Fig. 7 (a). The local strain mapping and image-based numerical simulation has been demonstrated to be complementary in revealing and understanding various deformation and fracture phenomena inside materials, especially those with inhomogeneous microstructures [44].

\subsection{Application of DVC}

The initial studies using DVC measurement of the strain field in a material [10] were not using synchrotron images. This is because DVC has been primarily applied to cellular materials, the microstructure of these being perfectly suited for such a measurement. DVC using standard laboratory tomography images of cellular materials has been used in [45] to analyze the deformation of rock wool and in [46] for sand. Fig. 8 is for example related to what has been presented in [45]. It shows, for this rock wool sample, a comparison between the spatial distribution of the regions undergoing high compressive strain (Fig. 8 (a)) and the regions exhibiting an initially low density (Fig. 8 (b)). The density is measured from the grey level in the reconstruction while the strain is measured using DVC. This comparison shows that the highly deformed regions are somehow related to the low density regions, at least in term of their orientation. In the scope of the present paper, the figure illustrates the capacity of DVC to analyze the distribution of strain in space. 
For standard bulk materials like the Al alloys mentioned earlier in this paper, the tracking procedure described in sections 2.1-2.5 is easier to implement than DVC because the number of particles is too small in the microstructure to serve as 'contrast support' for a correlation measurement. To overcome this problem, in Bornert et al. [47] we have fabricated $\mathrm{Al}$ based materials exhibiting a high amount of fine $\mathrm{Cu}$ particles used as markers. A minimum volume fraction of $4 \%$ of these particles was shown to be required to allow a precise DVC measurement. Note that this "marker" procedure has also been used in [8] with $\mathrm{W}$ particles for a strain measurement using the microstructural tracking method. Synchrotron DVC has more recently been used to study the deformation of polymer foams [11], rocks [48], the solidification of aluminium alloys [49] and the propagation of fatigue cracks in cast iron [50]. In all these cases, the intrinsic contrast of the microstructure in the X-ray tomography images was sufficient to serve as a support for the correlation procedure.

\section{Summary}

There are a few imaging techniques that can be nowadays used to visualize microstructural features of structural materials in 3D. Of these, the in-situ X-ray microtomography provides a unique possibility to access the time evolution behaviours of internal microstructure as well as its $3 \mathrm{D}$ character in high resolution. Advanced techniques for measuring local strain distribution have been readily enabled on the basis of the in-situ synchrotron radiation X-ray microtomography images with reasonable 
spatial resolution and a relatively large specimen size. The two main ones are microstructural tracking and DVC. These two different ways of measuring local strain fields induced by an external stimulus on the material has been described and illustrated in the present paper.

A successful development of these local strain mapping techniques has been achieved by combining with the advanced image processing techniques. Different algorithms can be merged into a sophisticated method making it possible to measure internal strain distribution with superior accuracy and reproducibility. This paves the way for transforming conventional metallurgical research approaches to more direct and accurate ones, since surface and cross-sectional observations reflect surface-specific 2D behaviour, which is often significantly different from that in the interior. More importantly, crystallographic deformation behaviours have also been clarified about the plastic deformation of polycrystalline materials by developing grain boundary tracking technique. This is helpful for us to get a down-to-earth understanding of practical materials. When this technique is combined with the 3D X-ray diffraction measurement, local strain variation in each grain can be interpreted in conjunction with the mismatch in crystallographic deformation behaviours among neighbouring grains. The local strain mapping technique would then be considered to be a more robust procedure that enables a comprehensive mechanical understanding of polycrystalline materials.

Considering the potential of local strain mapping in materials science and engineering, we believe that more widespread application of the technique will have a high impact on 
the understanding of various issues, such as thermomechanical processes and mechanical properties.

\section{Acknowledgments}

This work was partly undertaken with the support of a Grant-in-aid for Scientific Research from JSPS through subject No. 20246102. The support of the Light Metal Educational Foundation to HT is also gratefully acknowledged.

\section{References}

1 Martin, T. A., Christie, G. R., and Bhattacharyya, D. Composite Sheet Forming, Chapter 6. Composite Materials Series, Elsevier, 1997, 11, 217-245.

2 Narendran, N., Shukla, A., and Letcher, S. Determination of fracture parameters using embedded fibre-optic sensors. Engng Fract Mech 1991, 38, 491.

3 Mummery, P. M., and Derby, B. Damage assessment in particle-reinforced metal matrix composites using x-ray microtomography J. Mater. Science, 1994, 29, 5615.

4 Humphreys, F, J. The thermomechanical processing of Al---SiC particulate composites. Proc. EMAG-MICRO 89. IOP, Bristol, 1990, 465.

5 Buffière, J. Y., Maire, E., Cloetens, P., Lormand, G., and Fougères, R. Characterization of internal damage in a $\mathrm{MMC}_{\mathrm{p}}$ using $\mathrm{X}$-ray synchrotron phase contrast microtomography. Acta Mater 1999, 47, 1613-1625.

6 Toda, H., Sinclair, I., Buffière, J-Y., Maire, E., Connolley, T., Joyce, M., Khor, K. 
H., and Gregson, P. Assessment of the fatigue crack closure phenomenon in damage-tolerant aluminium alloy by in-situ high-resolution synchrotron X-ray microtomography. Phil. Mag. A. 2003, 83, 2429-2448

7 Toda, H., Sinclair, I., Buffière, J.Y., Maire, E., Khor, K. H., Gregson, P, and Kobayashi, T. A 3D measurement procedure for internal local crack driving forces via synchrotron X-ray microtomography. Acta Mater, 2004, 52, 1305.

8 Nielsen, S.F., Poulsen, H.F., Beckmann, F., Thorning, C., and Wert, J.A. Measurements of plastic displacement gradient components in three dimensions using marker particles and synchrotron X-ray absorption microtomography. Acta Materialia, 2003, 51, 2407-2415.

9 Kobayashi, M., Toda, H., Kawai, Y., Ohgaki, T., Uesugi, K., Wilkinson, D.S., Kobayashi, T., Aoki, Y., and Nakazawa, M. High-density three-dimensional mapping of internal strain by tracking microstructural features. Acta Materialia 2008, 56, 2167-2181.

10 Bay B., Smith T., Fyhrie D., and Saad M. Digital Volume Correlation: Threedimensional Strain Mapping Using X-ray Tomography, Experimetal Mechanics, 1999, 39, 218-227.

11 Roux, S., Hild, F., Viot, P., and Bernard, D. Three dimensional image correlation from X-Ray computed tomography of solid foam. Comp Part A., 2008, 39, 1253.

12 Hild, F., and Roux, S. Digital image correlation, from displacement measurement to identification of elastic properties - a review. Strain, 2006, 42, 69-80. 
13 Bay, B. Methods and applications of digital volume correlation. Strain Analysis, 2008, 43, 745-760.

14 Zhang, H., Toda, H., Qu, P. C., Sakaguchi, Y., Kobayashi, M., Uesugi, K., and Suzuki, Y. Three-dimensional fatigue crack growth behaviour in an aluminium alloy investigated with in situ high-resolution synchrotron X-ray microtomography. Acta Mater, 2009, 57, 3287-3300.

15 Qu, P. C., Toda, H., Zhang, H., Sakaguchi, Y., Qian, L., Kobayashi, M., and Uesugi, K. Local crack driving force analysis of a fatigue crack by a microstructural tracking method. Scripta Mater, 2009, 61, 489-492.

16 Uesugi, K., Suzuki, Y., Yagi, N., Tsuchiyama, A., and Nakano, T. Characterization of the sputtered-sliced zone plate for high energy X-raysNucl. Instr. And Meth., 2001, 467-468, 853-856.

17 Kobayashi, M., Toda, H., Uesugi, K., Ohgaki, T., Kobayashi, T., Takayama, Y., and Ahn, B-G. Preferential penetration path of gallium into grain boundary in practical aluminium alloy. Philos, Mag., 2006, 86(28), 4351-4366.

18 Leclere, D., Kobayashi, M., Toda, H., Uesugi, K., Suzuki, Y., Aoki, Y., and Nakazawa, M. Acta Materialia, 2011, to be submitted.

19 Toda, H., Uesugi, K., Takeuchi, A., Minami, K., Kobayashi, M., and Kobayashi, T. Appl Phys Lett., 2006, 89, 143112.

20 Suzuki, Y., Toda, H., and Schroer, C. Tomography using magnifying optics. Advanced tomographic methods in materials research and engineering, Oxford Univ. 
Press, Oxford, 2008, 181-210.

21 Mokso, R., Cloetens, P., Maire, E., Ludwig, W., and Buffière, J,-Y. Nanoscale zoom tomography with hard x rays using Kirkpatrick-Baez. Applied Physics Letters, 2007, 90, 144104.

22 Requena, G., Cloetens, P., Altendorfer, W., Poletti, C., Tolnai, D., Warchomicka, F., and Degischer, P. Sub-micrometer synchrotron tomography of multiphase metals using Kirkpatrick-Baez optics. Scripta Mater, 2009, 61(7), 760-763.

23 Toda, H., Yamamoto, S., Kobayashi, M., and Uesugi, K. Direct measurement procedure for three-dimensional local crack driving force using synchrotron X-ray microtomography. Acta Materialia, 2008, 56, 6027-6039.

24 Toda, H., Ohgaki, T., Uesugi, K., Kobayashi, M., Kuroda, N., Kobayashi, T., Niinomi, M., Akahori, T., Makii, K., and Aruga, Y. Quantitative assessment of microstructure and its effects on compression behaviour of aluminium foams via high-resolution synchrotron X-ray tomography. Metall. Mater. Trans. A. 2006, 37A (4), 1211-1220.

25 Toda, H., Hidaka, T., Kobayashi, M., Uesugi, K., Takeuchi, A., and Horikawa, K. Growth behaviour of hydrogen micropores in aluminium alloys during hightemperature exposure, Acta Materialia, 2009, 57, 2277-2290.

26 Buffiere, J-Y., Maire, E., Adrien, J., Masse, J-P., and Boller, E. In Situ Experiments with $\mathrm{X}$ ray Tomography: An Attractive Tool for Experimental Mechanics. Experimental Mechanics, 2010, 50(3), 289-305. 
27 Qian, L,. Toda, H., Uesugi, K., Kobayashi, M., and Kobayashi, T. Direct Observation and Image-Based Simulation of Three-Dimensional Tortuous Crack Evolution inside Opaque Materials. Physical Review Letters, 2008, 100, 115505.

28 Toda, H., Nishimura, T., Uesugi, K., Suzuki, Y., and Kobayashi, M. Influence of high-temperature solution treatments on mechanical properties of an $\mathrm{Al}-\mathrm{Si}-\mathrm{Cu}$ aluminium alloy. Acta Materialia, 2010, 58, 2014-2025.

29 Toda, H., Minami, K., Koyama, K., Ichitani, K., Kobayashi, M., Uesugi, K., and Suzuki, Y. Healing behaviour of pre-existing hydrogen micropores in aluminium alloys during plastic deformation. Acta Materialia, 2009, 57, 4391-4403.

30 Lourenco, L., Recent Advances in LSV, PIV and PTV, in: Flow Visualization and Image Analysis, ed. F.T.M. Nieuwstadt, Kluwer Academic Publishers, 1993, 81-89L.

31 Lorensen, W. E., and Cline, H. E. Marching cubes: A high resolution 3D surface construction algorithm. Computer graphics (ACM) 1987, 21, 163-169.

32 Okamoto, K., Hassan, Y.A., and Schmidt, W. D. New tracking algorithm for particle image velocimetry, Experimental Fluids, 1995, 19(5), 324-347.

33 Kobayashi, M., Toda, H., Kawai, Y., Nakazawa, M., Aoki, Y., Uesugi, K., and Suzuki, Y. Three-dimensional Plastic Strain Mapping Technique for X-ray Microtomography Proc. ICAA-12, Yokohama, Japan, J. Inst. Light Met., 2010, 1330-1335.

34 Nakazawa, M., Kobayashi, M., Toda, H., and Aoki, Y. Proposal of a method to analyze 3D deformation/fracture characteristics inside materials based on a stratified matching approach. MACHINE VISION AND APPLICATIONS, 2010, 21(5), 687- 
694.

35 Rosenfeld, A., Hummel, R, A., and Zucker, S, W. Scene labelling by relaxation operation. IEEE Trans. Syst. Man Cybern. 1976, 6, 420-433.

36 Barber, C. B., Dobkin, D. P., and Huhdanpaa, H. T. The Quickhull Algorithm for Convex Hulls. ACM Transactions on Mathematical Software, 1996, 22, 469-483.

37 Weckenmann, A. and Krämer, P. Proc. XIX IMEKO World Congress, Fundamental and Applied Metrology, Portugal, 2009, 1888-1892.

38 Toda, H., Yamaguchi, T., Nakawaza, M., Aoki, Y., Uesugi, K., Suzuki, Y., and Kobayashi, M. Four-Dimensional Annihilation Behaviours of Micro Pores during Surface Cold Working Mater. Trans., 2010, 51(7), 1288-1295.

39 Kobayashi, M., Toda, Uesugi, K., and Suzuki, Y. Acta Materialia, to be submitted.

40 Ludwig, W., and Bellet, D. Penetration of liquid gallium into the grain boundaries of aluminium: a synchrotron radiation microtomographic investigation, Materials Science and Engineering A, 2000, 281, Issues 1-2, 198-203,

41 King, A., G, Johnson, D. Engelberg, W. Ludwig, and Marrow, J. Observations of Intergranular Stress Corrosion Cracking in a Grain-Mapped Polycrystal. Science 18 July 2008 321, 382-385,

42 Fu, X., Poulsen, H,F., Schmidt, S., Nielsen, S, F., Lauridsen, E, M., and Jensen, D. Non-destructive mapping of grains in three dimensions. Scripta Mater, 2003, 49(11), 1093-1096

43 Toda, H., Kobayashi, M., Suzuki, Y., Takeuchi, A., and Uesugi, K. Journal of the 
Japanese Society for Non-Destructive Inspection, 2009, 58(10), 433-438 (in Japanese)

44 Toda, H., Takata, M., Ohgaki, T., Kobayashi, M., Kobayashi, T., Uesugi, K., Makii, K., and Aruga, Y. 3-D Image-Based Mechanical Simulation of Aluminium Foams: Effects of Internal Microstructure. Adv. Engng. Mater. 2006, 8(6), 459-467

45 Hild, F., Maire, E., Roux, S., and Witz, J. F. Three dimensional analysis of a compression test on stone wool. Acta Materialia 2009, 57, 3310-3320.

46 Hall, S, A., Bornert, M., Desrues, J., Pannier, Y., Lenoir, N., Viggiani, G., and Besuelle, P. Discrete and continuum analysis of localised deformation in sand using $\mathrm{X}$-ray $\mu \mathrm{CT}$ and volumetric digital image correlation. Géotechnique, 60, 5, 315 -322.

47 Bornert, M., Chaix, J, M., Doumalin, P., Dupré, J, C., Fournel, T. D., Jeulin, Maire, E., Moreaud, M., and Moulinec, H. Mesure tridimensionnelle de champs cinématiques par imagerie volumique pour l'analyse des matériaux et des structures. Instrumentation, Mesure, Métrologie, In french. 2004, 88, 43.

48 Lenoir, N., Bornert, M., Desrues, M., Besuelle, P., and Viggiani, G. Volumetric Digital Image Correlation Applied to X-ray Microtomography Images from Triaxial Compression Tests on Argillaceous Rock. Strain 2007, 43, 193.

49 Terzi, S., Salvo, L., Suery, M., Limodin, N., Adrien, J., Maire, E., Pannier, Y., Bornert, M., Bernard, D., Felberbaum, M., Rappaz, and Boller, M, E. In situ Xray tomography observation of inhomogeneous deformation in semi-solid aluminium alloys, Scripta Materialia, 2009, 61(5), 449-452, 
50 Limodin, N., Réthoré, J., Buffière, J-Y., Gravouil, A., Hild, F., and R, Stéphane.

Crack closure and stress intensity factor measurements in nodular graphite cast iron using three-dimensional correlation of laboratory X-ray microtomography images. Acta Mater. 2009, 57(14), 4090-4101

Fig.1 A schematic illustration of the local strain mapping process that consists of image registration, particle tracking during two consecutive loading steps and strain calculation.

Fig 2 2D schematic description of the principle of Digital Volume Correlation showing the initial image $f(x)$ and the deformed image $g(x)$. The displacement of two recognizable patterns can be retrieved by correlation of subvolumes.

Fig.3 Fig. 3 Measured 3D local strain distributions within a cubic specimen during the monotonic compression of an $\mathrm{Al}-4 \% \mathrm{Mg}$ alloy. $\mathrm{Pb}$ particles were artificially dispersed in order for the local strain measurement. Strain distribution has been measured between the macroscopic strain levels described below each figure. Specimen dimensions are expressed in $\mu \mathrm{m}$.

Fig. 4 3D perspective views (left images) of a pore together with local strain distributions shown on a virtual cross-section (right images), representing the spatial distributions of accumulated equivalent plastic strain and its correspondence with pore annihilation behaviors during the surface cold working of a cast $\mathrm{Al}-6.9 \% \mathrm{Si}$ $0.4 \% \mathrm{Mg}$ alloy. 
Fig. 5 Relationships between shrinkage behaviors of pores and local effective strain around pores, representing the dominant factor for pore closure. Note that open symbols are monotonic compression and solid marks are the data for surface cold working.

Fig. 6 A schematic illustration of the grain boundary tracking technique.

Fig. 7 Comparison between an image-based numerical simulation and experiments during the monotonic loading of an Al-7Si-1Mg alloy. (a) A subvolume extracted from an entire tomographic volume; and (b) an equivalent stress contour in an identical subvolume.

Fig. 8 3D view of the spatial distribution of the regions with a low initial density for the rock wool material in ref [45] is shown in (a), together with that of the regions with a high local compressive strain (the same rock wool sample and location with (a)) in (b). The compression axis is vertical in the figure and the size of one side of the observed volume is $30 \mathrm{~mm}$. 


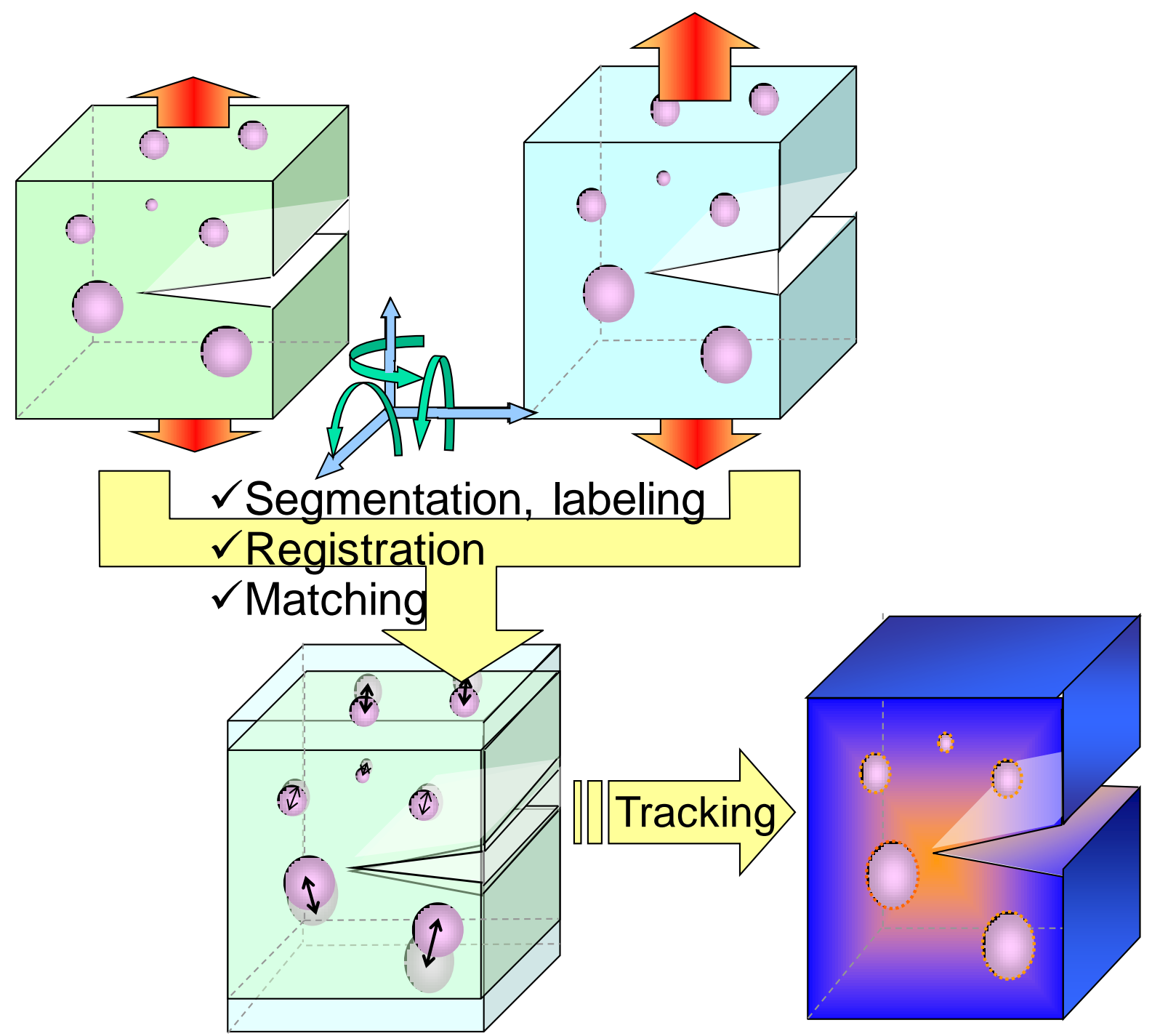

Fig. 1 A schematic illustration of the local strain mapping process that consists of image registration, particle tracking during two consecutive loading steps and strain calculation. 


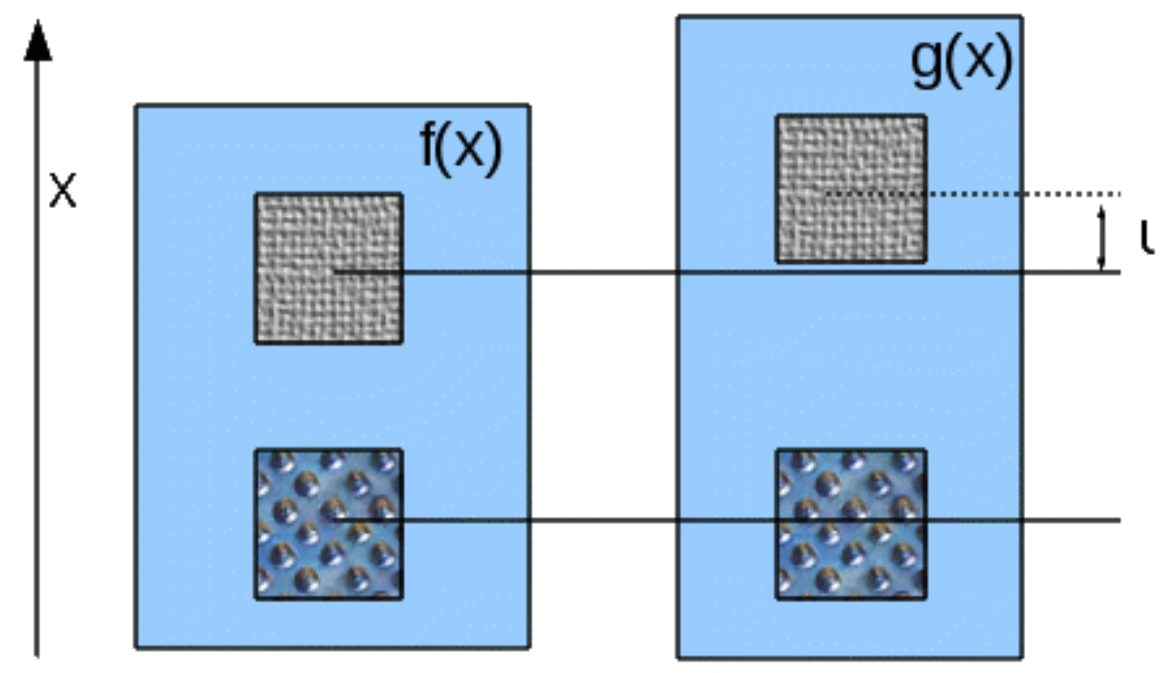

Fig 2 2D schematic description of the principle of Digital Volume Correlation showing the initial image $\mathrm{f}(\mathrm{x})$ and the deformed image $\mathrm{g}(\mathrm{x})$. The displacement of two recognizable patterns can be retrieved by correlation of subvolumes. 


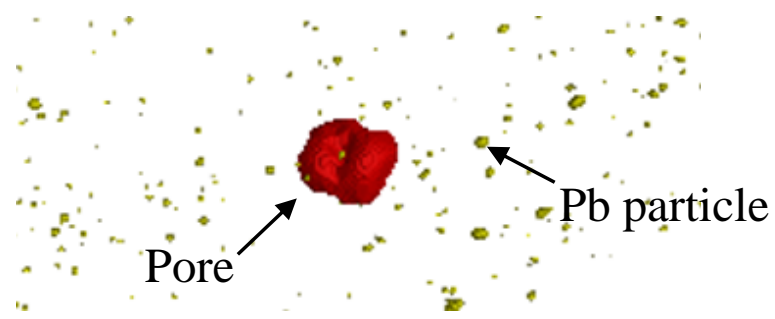

(a) Before hardening
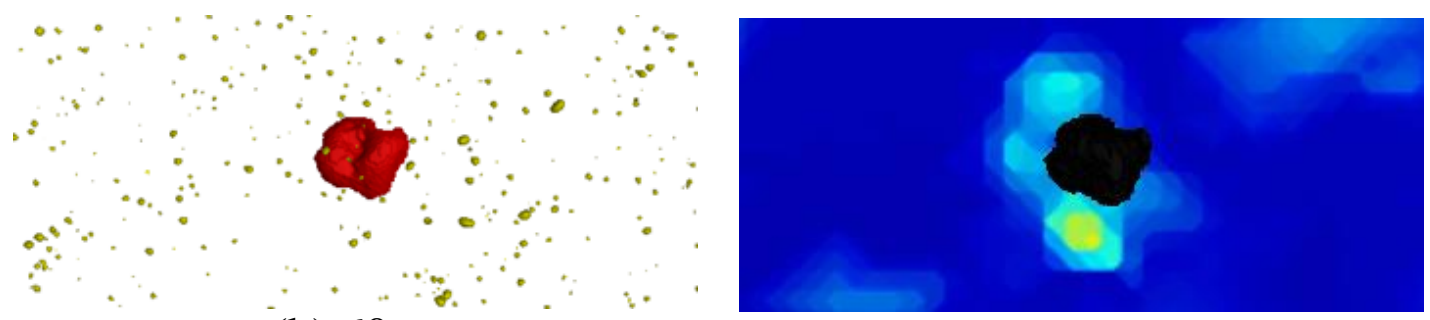

(b) $60 \mathrm{sec}$
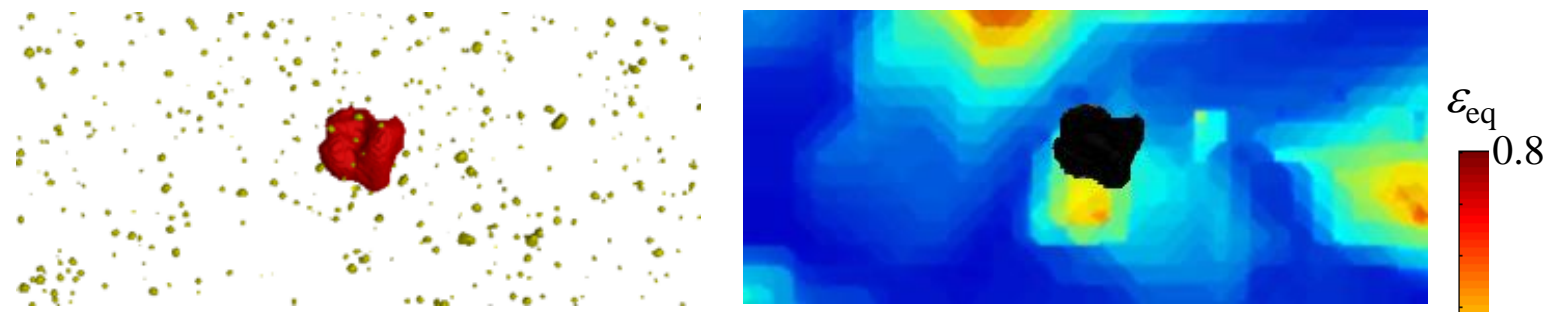

(c) $240 \mathrm{sec}$
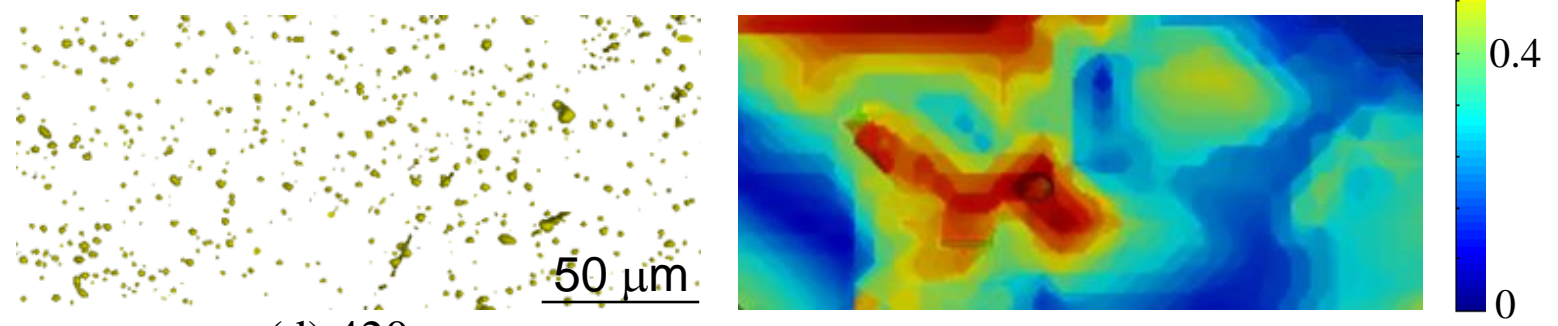

(d) $420 \mathrm{sec}$

Fig. 4 3D perspective views (left images) of a pore together with local strain distributions shown on a virtual cross-section (right images), representing the spatial distributions of accumulated equivalent plastic strain and its correspondence with pore annihilation behaviors during the surface cold working of a cast $\mathrm{Al}-6.9 \% \mathrm{Si}-0.4 \% \mathrm{Mg}$ alloy. 


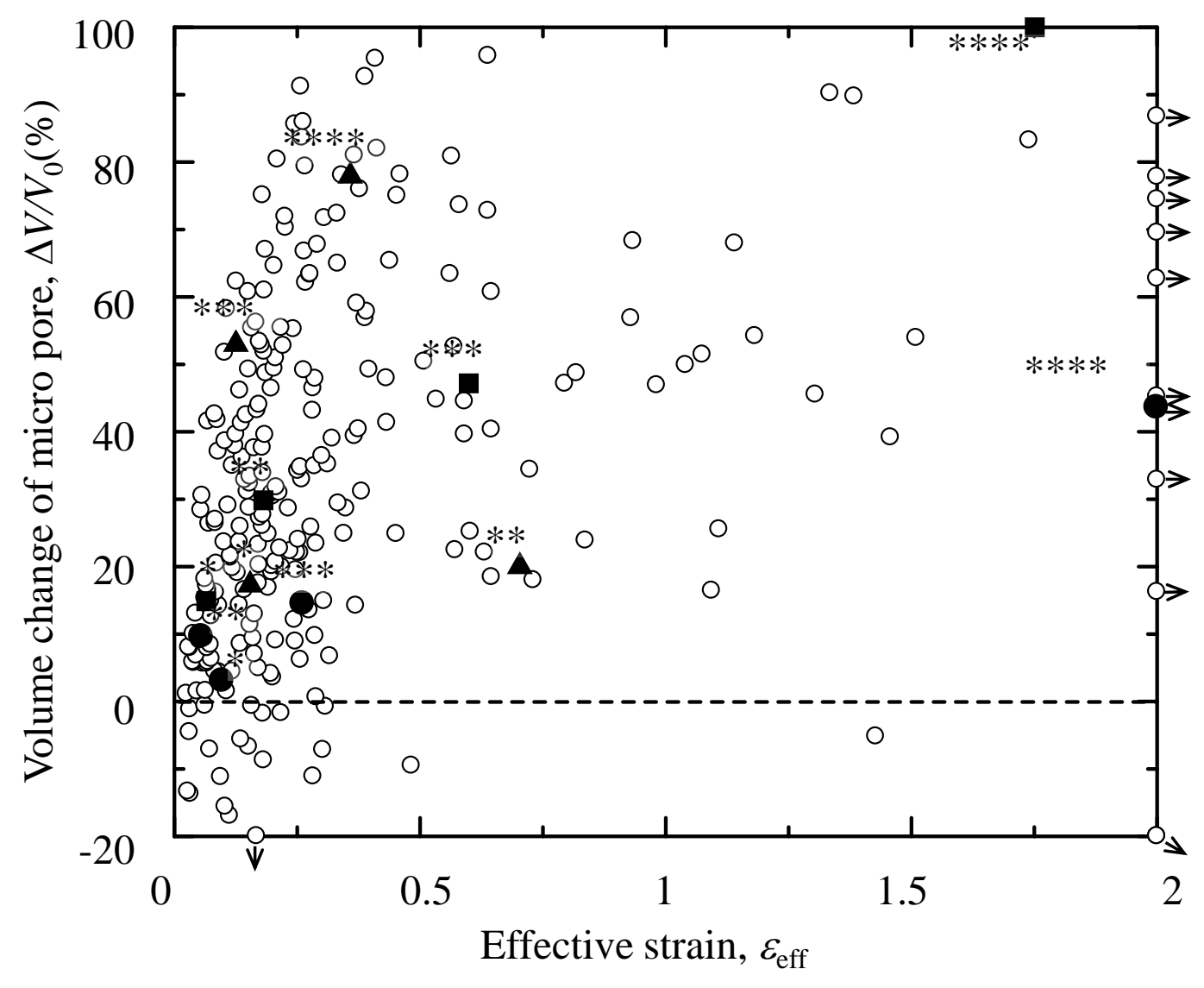

Fig. 5 Relationships between shrinkage behaviors of pores and local effective strain around pores, representing the dominant factor for pore closure. Note that open symbols are monotonic compression and solid marks are the data for surface cold working. 


\section{Load $1 \quad$ Load 2}

In-situ observation during deformation

Inverse tracking of each particle
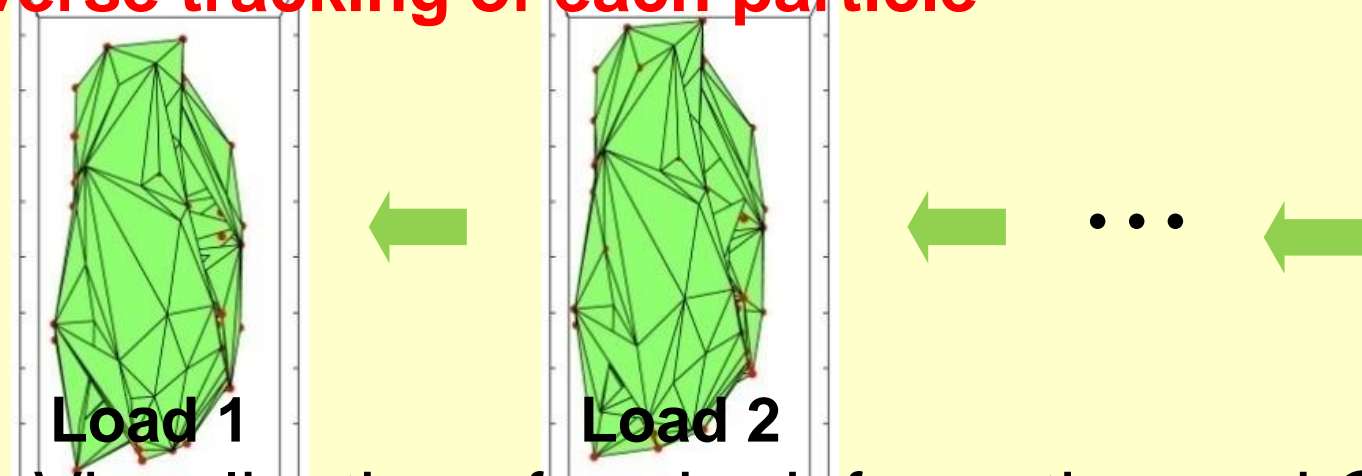

Final load

Final load

(GB visualized)

Visualization of grain deformation and GB stress/strain

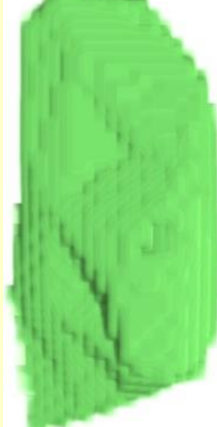

Load 1

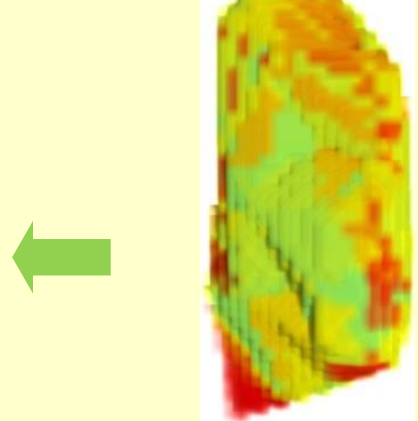

Load 2

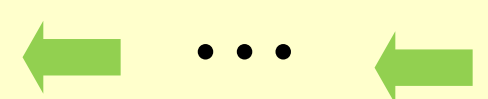

Visualization of stress/strain inside each grain

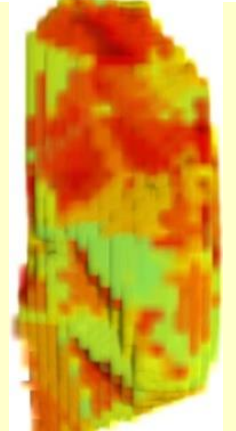

Final load
Classification into ...

GB particles

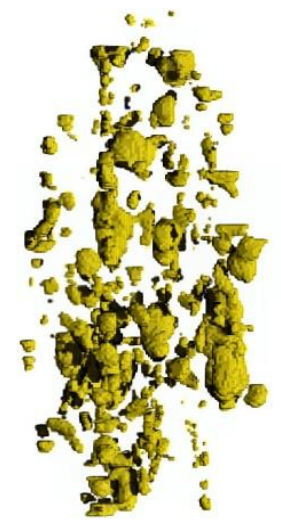

Particles in grain interior

Fig. $6 \mathrm{~A}$ schematic illustration of the grain boundary tracking technique. 


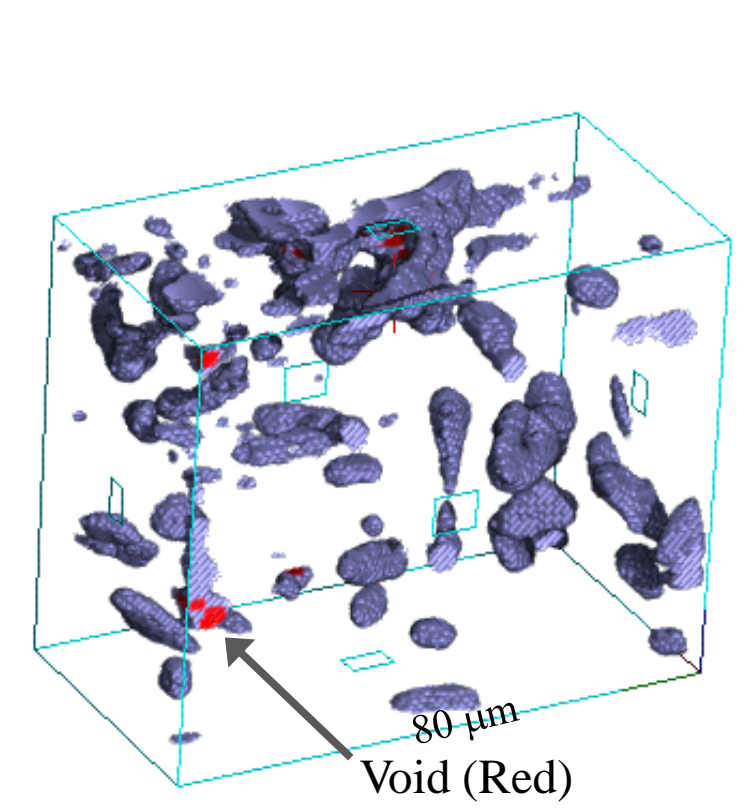

(a) In-situ observation

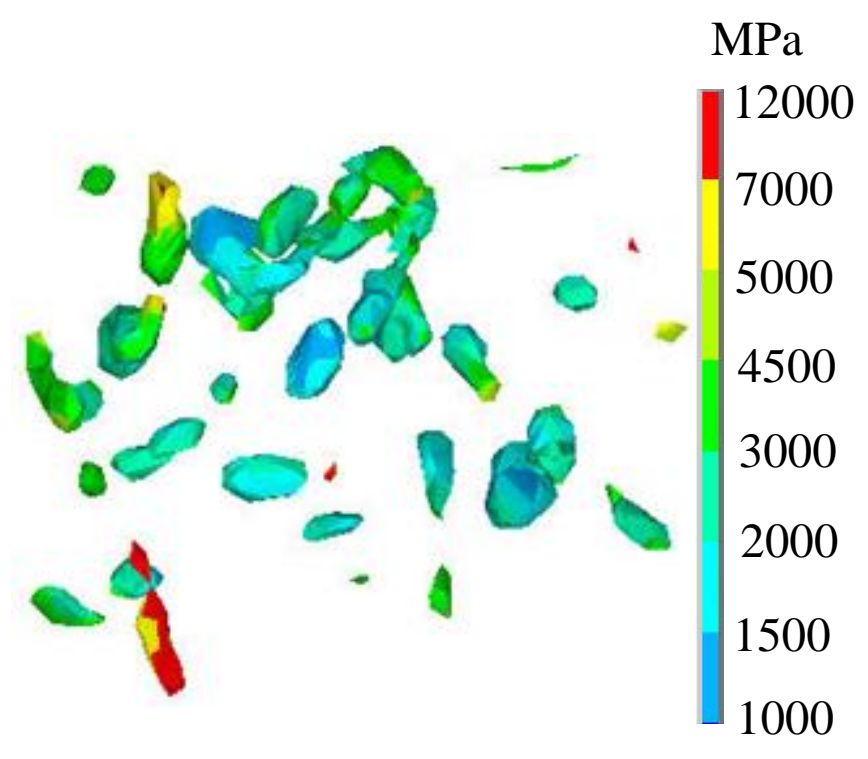

(b) Image-based simulation

Fig. 7 Comparison between an image-based numerical simulation and experiments during the monotonic loading of an $\mathrm{Al}-7 \mathrm{Si}-1 \mathrm{Mg}$ alloy. (a) A subvolume extracted from an entire tomographic volume; and (b) an equivalent stress contour in an identical subvolume. 

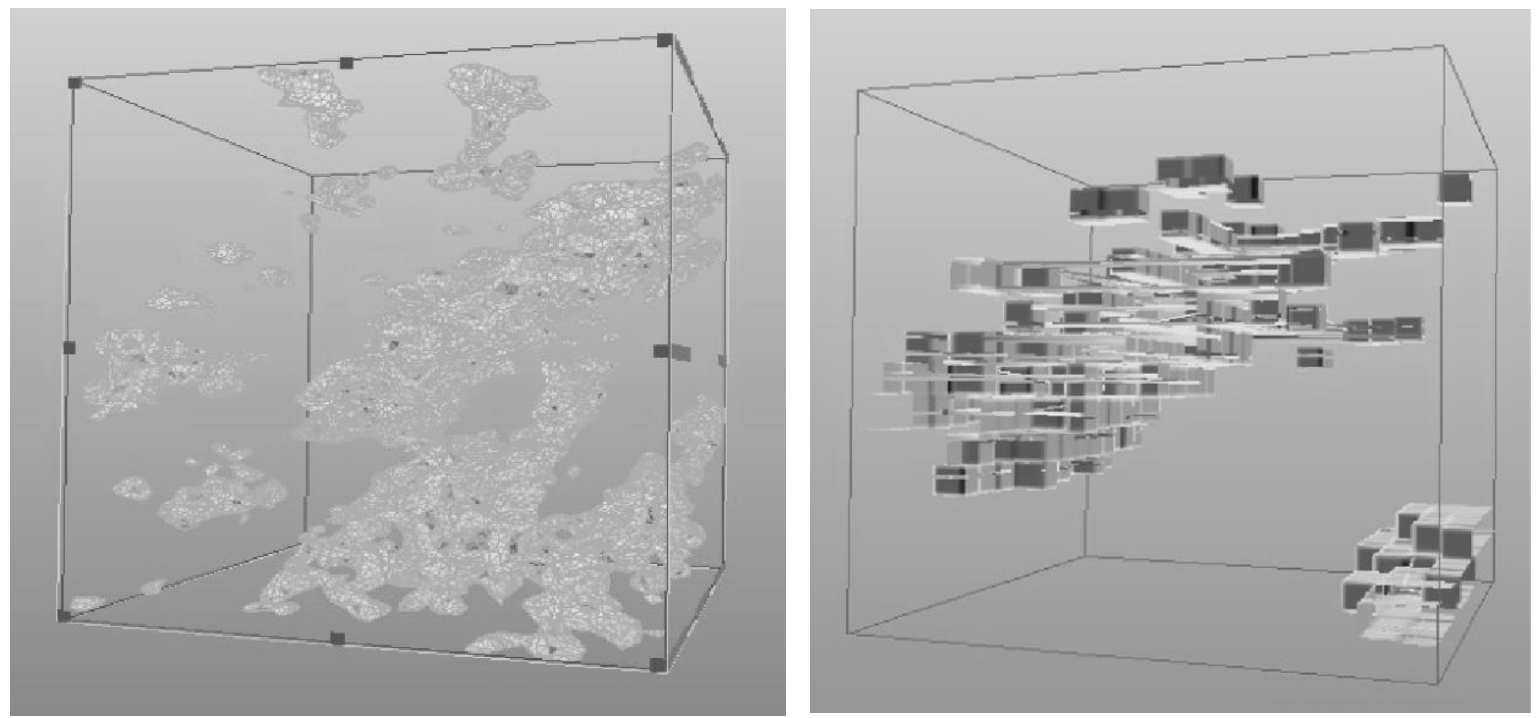

Fig. $83 \mathrm{D}$ view of the spatial distribution of the regions with a low initial density for the rock wool material in ref [45] is shown in (a), together with that of the regions with a high local compressive strain (the same rock wool sample and location with (a)) in (b). The compression axis is vertical in the figure and the size of one side of the observed volume is $30 \mathrm{~mm}$. 